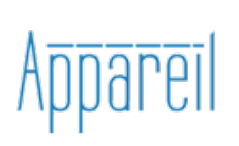

Appareil

$7 \mid 2011$

La transparence

\title{
Révolution française, opinion publique et transparence : les fondements de la démocratie moderne
}

Philippe Münch

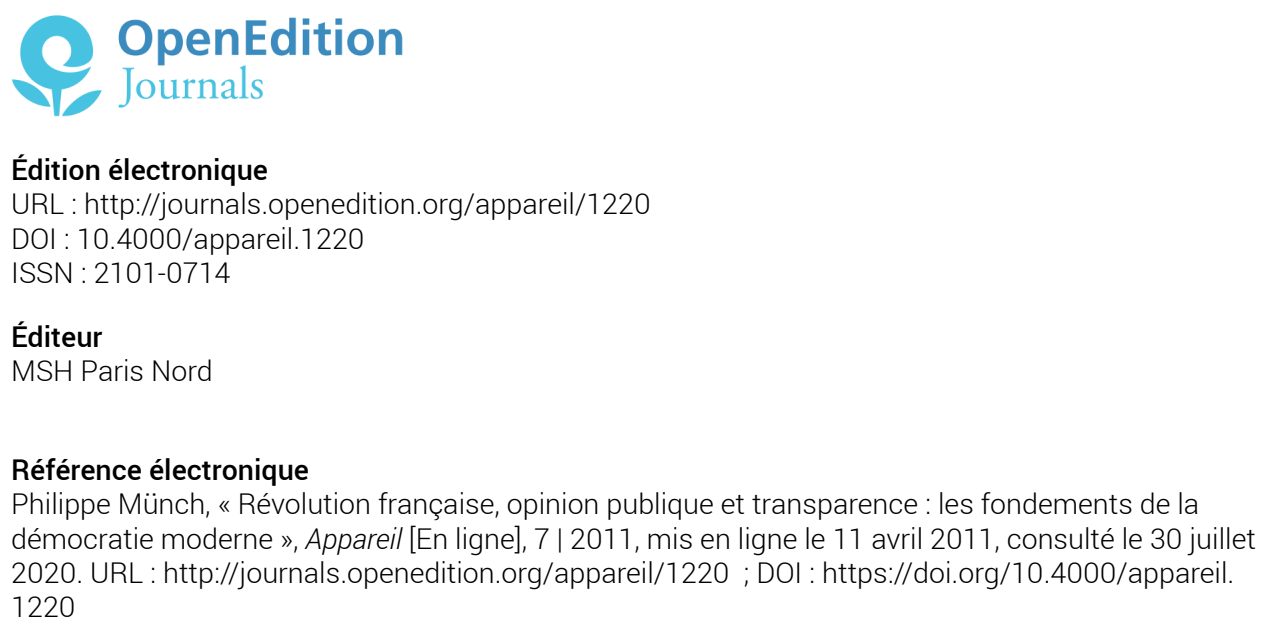

Ce document a été généré automatiquement le 30 juillet 2020.

\section{(c) (1) ()}

Appareil est mis à disposition selon les termes de la Licence Creative Commons Attribution - Pas d'Utilisation Commerciale - Pas de Modification 4.0 International. 


\title{
Révolution française, opinion publique et transparence : les fondements de la démocratie moderne
}

\author{
Philippe Münch
}

1 La démocratie française naît en 1789 en opposition à la politique absolutiste des rois de France fondée sur le secret, la domination et la seule volonté du monarque ${ }^{1}$. Pour les révolutionnaires, la question de la transparence, abordée à travers les concepts d' opinion publique et de publicité, est absolument centrale au projet démocratique, qui vient saper les fondements de la monarchie absolue. Depuis les trente dernières années, tout un pan de l'historiographie s'est tourné vers cette problématique féconde, comme en témoigne d'ailleurs le colloque La Révolution française au carrefour des recherches, qui a présenté le bilan de la recherche post-Bicentenaire ${ }^{2}$.

2 De ce vaste chantier découle plusieurs polémiques théoriques que nous ne pourrons pas aborder dans cet article, faute d'espace. Nous retiendrons néanmoins deux thèses opposées en apparence mais qui dévoilent la double trame de la modernité. D'un côté, il y a celle de Jürgen Habermas défendue dans son ouvrage devenu classique : L'espace public ${ }^{3}$. La sphère publique, qui apparaît en France au milieu du xvIII ${ }^{e}$ siècle, est définie comme un espace, soustrait de l'emprise de l'État et de l'Église, où des personnes privées font un usage public de la raison, au sens kantien. Cet espace, qui se constitue essentiellement avec l'avènement de la société bourgeoise, permet l'éclosion d'une nouvelle sociabilité articulée autour de valeurs en opposition avec l'univers aristocratique et absolutiste : la raison, l'égalité, la publicité.

Par le biais de la pensée d'Augustin Cochin, François Furet a proposé une lecture différente mais qui repose néanmoins sur le même postulat de départ : l'émergence, à partir de 1750, d'une sociabilité démocratique composée de sociétés de pensée (salons, cafés, loges, etc.), qui développe un nouveau mode de relations entre les citoyens et le pouvoir ${ }^{4}$. Cette sociabilité est qualifiée de démocratique dans la mesure où elle est fondée 
sur l'égalité abstraite entre les individus. En cela, elle est construite en opposition à la société traditionnelle organisée autour d'ordres et d'états régis par les principes de hiérarchie et de privilèges. Jusque-là, et si on évacue la problématique de la bourgeoisie et du capitalisme ${ }^{5}$, l'approche de F. Furet rejoint la thèse de J. Habermas sur la politisation de la sphère littéraire et la constitution d'un espace contraire aux logiques absolutistes de la monarchie française.

4 L'approche furetienne prend toutefois une tangente différente sur la nature de l'espace qui est constitué en tant que tel. Chez Furet - Cochin, les sociétés de pensée sont des lieux de formulation d'opinion commune, de fabrication de consensus où se dessine la volonté générale de Rousseau'. Le jacobinisme représente la forme achevée des sociétés de pensée, de cette machine de socialisation qui produit du consensus et de la vérité, par laquelle on exclut et on épure. Chez J. Habermas, la nouvelle sociabilité crée au contraire un espace critique, où l'on fait un usage public de la raison et de son jugement. Le sens accordé à l'opinion publique et à la transparence est alors différent, évoquant la pluralité et la liberté de la pensée et non l'unanimité. Cet espace renvoie, en quelque sorte, à ce que Hannah Arendt décrit comme les fondements du politique, où les hommes discutent et décident en commun?.

Les deux perspectives peuvent donc sembler irréconciliables dans leur finalité. Mais à situer cette tension au cœur même de la nouvelle culture politique, le fossé peut apparaître moins grand. L'émergence de l'opinion publique au $\mathrm{XVIII}^{\mathrm{e}}$ siècle renverrait à deux phénomènes distincts mais enchevêtrés, qui créent une tension au sein de la démocratie naissante. Le principal objectif de cet article est de montrer que F. Furet et $\mathrm{J}$. Habermas disent une part de vérité sur la nature de la sociabilité démocratique du XVIII ${ }^{\mathrm{e}}$ siècle. Elle marque autant la libération et la prolifération de la parole, d'une parole désormais publique et non plus secrète, que le résultat d'un processus qui tend à fabriquer de l'unanimité par l'exclusion. La démocratie serait ainsi animée par un double processus, consubstantiel à cette forme politique : un premier processus qu'on pourrait qualifier de libéral, favorisant une extension de l'espace public, et un autre plus sombre, de nature anti-démocratique, qui produit du consensus en resserrant les limites de la sphère publique. C'est la double trame de la modernité que nous tenterons d'analyser'.

6 Pour en faire la démonstration, nous utiliserons une méthode d'analyse qui s'inspire de l'histoire non quantitative d'Antoine de Baecque". Elle propose une "stratégie de l'araignée ", c'est-à-dire la confection par l'historien d'une toile interprétative qui, à défaut d'être totalement systématique et quantifiée, rend intelligible les composantes de l'objet d'étude, soit la nouvelle culture politique démocratique. Cette démarche interprétative appelle une lecture de sources hétérogènes: discours d'assemblée, journaux, brochures, pamphlets. Isolée, une source est pauvre nous dit A. Baecque. Regroupées, croisées et comparées, elles dévoilent à l'historien toute leur potentialité. L'interprétation se peaufine à travers cet univers intertextuel.

7 Notre article sera divisé en trois parties. La première partie aura comme objectif de montrer que l'opinion publique suppose à la fois une forme de pouvoir et un espace de liberté. La deuxième partie tentera de mettre en évidence l'idée que l'opinion publique évoque un idéal de communauté politique fondée sur la transparence et la publicité. Enfin, il s'agira d'analyser les problèmes relatifs à la pratique de la dénonciation qui, censée assurer la transparence de l'espace public, devient rapidement un instrument d'exclusion au nom du salut public. 


\section{Pouvoir, souveraineté et liberté}

Les événements de 1789 ont rapidement été perçus et interprétés par les acteurs de l'époque comme un moment de fondation politique qui aurait provoqué une véritable rupture avec le passé, avec ce qui sera appelé l'Ancien Régime. Tocqueville nous a mis en garde contre cette lecture rapide traçant une ligne de partage historique entre un avant et un après 1789 , qui découle du constructivisme révolutionnaire, c'est-à-dire de cette volonté de reconstruire toute la société française (tabula rasa) : «La France n'a point déposé autour d'elle les germes de la Révolution, elle y a développé ceux qui y étaient déjà ; elle n'a point été le dieu qui crée, mais le rayon de soleil qui fait éclore ${ }^{10}$ " C'est le cas notamment avec le concept d'opinion publique qui était largement véhiculé par les philosophes à travers la République des Lettres. Les révolutionnaires sont donc en quelque sorte les héritiers d'une culture politique née sous Louis XV, autour de 1750, mais des héritiers qui auront mené à son terme la portée normative de l'idée d'opinion publique, construite en opposition avec la politique absolutiste de la monarchie menée depuis Louis XIV ${ }^{11}$.

9 L'opinion publique représente une figure centrale de la politique révolutionnaire de refondation. À travers elle se pense notamment les nouveaux droits des citoyens, qui se trouvent au cœur du projet démocratique de 1789. En effet, elle renvoie à deux articles fondamentaux de la déclaration des droits de l'homme : soit la souveraineté populaire (art. 3) et la liberté de la presse (art. 11).

10 L'opinion publique est pensée comme la source légitime de la nation; les deux termes sont en relation étroite dans le discours révolutionnaire. Jacques Guilhaumou a montré qu'à la veille de la Révolution, elle est constamment associée à l'« engouement de la nation », à ses « forces ", à ses « vœux » et à son « désir général ${ }^{12}$ ». Les députés Volney, Pétion, Target, Thouret et Malouet l'assimilent plus particulièrement à la volonté générale ${ }^{13}$. Le monarchien Bergasse défend une conception assez proche en la considérant comme le véritable "produit de toutes les intelligences et de toutes les volontés; on peut la regarder en quelque sorte comme la conscience manifestée d'une nation entière ${ }^{14}$. Pour le journal Révolutions de Paris, l'opinion publique constitue, dans le même sens, «la déclaration inaltérable de la volonté générale » (été 1789). Dans son " Discours sur la liberté de la presse », prononcé à la société des Jacobins le 11 mai 1791, Robespierre associe les deux termes à plusieurs reprises ${ }^{15}$.

11 Cette relation sémantique montre bien que la nation s'incarne souverainement dans l'opinion publique. Les représentants de la nation doivent ainsi se laisser guider par elle. Les législateurs ne sont que "l'organe» de l'opinion publique soutient le révolutionnaire Robert ${ }^{16}$. Durant le débat sur la déchéance du roi à l'été 1792 , Brissot a ces paroles particulièrement instructives qui méritent d'être citées : "Qui êtes-vous, Messieurs? les représentants d'un grand peuple. Où est votre force? dans le peuple. Quand vous soutient-elle? lorsque vous rendez des décrets sages \& justes. Comment pouvez-vous être certains de rendre de pareils décrets? lorsque vous consultez l'opinion publique, lorsque vous ne faites, pour ainsi dire, que l'énoncer [...]» Le pouvoir législatif, qui tire sa légitimité du peuple, ne doit être que le prolongement de cette opinion considérée comme souveraine.

12 En fait, l'opinion publique a tous les attributs du pouvoir souverain. En plus d'incarner la nation et la volonté générale, donc des forces anonymes et universelles, on parle 
d'elle comme la «reine du monde ». Elle a un «empire ». C'est aussi un «tribunal ${ }^{17}$ » qui juge les hommes, comme l'avait bien dit Malesherbes en 1775 à l'Académie française ${ }^{18}$. Elle est dotée également d'une puissance irrésistible. Avant la Révolution, Mercier parlait déjà, dans les Tableaux de Paris, d'une force à laquelle on ne peut " résister ${ }^{19}$ ». Elle est perçue comme une véritable puissance qui finit par s'imposer nécessairement aux hommes. Produite par eux, elle échappe pourtant à la volonté humaine. En ce sens, Pétion dit, dans son Discours sur la liberté de la presse (1791), «Et d'ailleurs n'est-il pas hors de la puissance humaine d'enchaîner l'opinion? On peut en suspendre, mais non pas en arrêter le cours. » On ne peut échapper au tribunal et à son jugement : «Hommes publics! consentez donc à être jugés aujourd'hui, puisque aussi vous le ferez demain ${ }^{20}$.» Pour Robespierre, elle est ni plus ni moins une «force invincible » : « le despotisme est contraint de reculer à chaque pas ou de venir se briser contre la force invincible de l'opinion publique et de la volonté générale ${ }^{21} »$. Dans le contexte du débat sur la guerre, en hiver 1792, l'homme de loi Machenaud rappelle à ses collègues jacobins que c'est l'opinion qui a fait la Révolution: «N'oublions jamais que ce n'est pas la force qui fait les révolutions et qui soutient les états, mais l'opinion. Reine du monde, son empire est irrésistible ${ }^{22}$ ». Elle est un «torrent » selon l'auteur de la Cabale des ministres, auquel on ne peut résister, car « le peuple seul est souverain [,] sa volonté seule fait la loi ${ }^{23}$.»

Dans le discours révolutionnaire s'impose donc une triade notionnelle à partir de laquelle se constituent la démocratie et le nouveau pouvoir populaire : souveraineté nation - opinion publique. Cet imaginaire de l'autorité, calqué en réalité sur le pouvoir de la monarchie absolue ${ }^{24}$, rend possible l'émergence d'un espace public, extérieur au pouvoir institutionnel, de même que l'essor de la liberté de penser et de dénoncer. De fait, en plus d'être une figure de pouvoir, l'opinion publique sous-tend aussi un principe de publicité. Le droit de publier ses opinions, c'est-à-dire de faire un usage public de sa raison selon la formule kantienne, découle de la valorisation de cette idée, qui sera reconnue par l'article 11 de la Déclaration des droits de l'homme: «La libre communication des pensées et des opinions est un des droits les plus précieux de l'homme $[. . .]^{25} »$ L'opinion publique suppose donc la libération de la parole et de l'écrit.

L'acte de dénoncer constitue une extension de ce droit ${ }^{26}$. On peut ainsi lire dans le Mercure de France que "la liberté de la presse avoit déféré au Peuple le pouvoir censorial. Les actions et les opinions, les abus et les projets lui sont exposés, son tribunal examine et juge ${ }^{27}$. " Les journalistes patriotes luttent, comme le soutient A. de Baecque, pour inclure l'acte de dénonciation dans les droits fondamentaux du citoyen ${ }^{28}$. Dans son journal le Patriote français, Brissot insiste sur le lien entre la liberté de la presse et la dénonciation : «[...] la liberté de la presse n'est-elle pas le seul moyen pour le Peuple de surveiller, d'éclairer, de censurer ses Représentants ${ }^{29}$. » Lors du débat sur la liberté de la presse à l'Assemblée nationale, Robespierre soutient que « le seul frein efficace des abus de l'autorité, c'est l'opinion publique [...]» De ce contre-pouvoir découle le droit et «la liberté de manifester son opinion individuelle sur la conduite des fonctionnaires publics ${ }^{30} »$. C'est le seul moyen, selon les révolutionnaires, d'assurer la liberté publique. Le droit de dénoncer est finalement reconnu par la Constitution de 1791: "La censure sur les actes des Pouvoirs constitués est permise " (chapitre V, art. 17) ${ }^{31}$. On accorde ainsi le droit de se méfier et de défier un pouvoir qui peut commettre des actes injustes bafouant les principes de la Révolution et les droits de l'homme. 

$\mathrm{du}$ concept d'opinion publique, de trouver un tiers-pouvoir, un intermédiaire, qui puisse concilier la souveraineté du peuple et la représentation ${ }^{32}$, ce que Rousseau considérait comme impossible ou antinomique. Or, la démocratie d'opinion le permettrait. La censure donne au peuple la possibilité de participer directement à la vie politique et de défendre lui-même la liberté et l'égalité. La pensée du démocrate Bonneville, bien étudiée par R. Monnier, est ici très significative puisqu'elle lie le droit de censure à l'opinion publique comme forme de pouvoir supérieur :

Le peuple, qui est tout, ne peut exercer par soi-même ni la puissance législative, ni la puissance exécutive ; mais un peuple aurait cessé d'être libre ou ne le serait pas encore, si un seul de ses membres était privé de son droit de censure, qui forme l'opinion publique, pondératrice de tous les différens pouvoirs ${ }^{33}$.

C'est parce qu'elle est une émanation de la puissance souveraine du peuple que l'opinion publique constitue en fait ce pouvoir suprême. Bonneville l'affirme pleinement dans La Bouche de Fer: ce pouvoir d'opinion «en ce qu'il appartient également à tous les individus, en ce que les individus peuvent l'exercer par eux-mêmes, sans représentation et sans danger pour le corps politique, constitue essentiellement la souveraineté nationale ${ }^{34}$.» Au-dessus de la loi produite par le corps législatif, il existerait donc une autre loi, plus puissante et souveraine. Le député Duport défend, en mai 1791, une idée similaire à la société des Jacobins : « [...] un grand peuple ne pouvant exercer lui-même sa souveraineté, et étant obligé de la déléguer, il doit se réserver un moyen de relever les fautes et les erreurs des hommes publics à qui il a donné le pouvoir de le lier lui-même.» Et d'ajouter que «sans la liberté la plus étendue de manifester ses opinions, il n'est point de gouvernement légitime ${ }^{35}$. »

\section{Liberté, transparence et démocratie}

17 Pensée comme une source d'autorité, souveraine, justicière et puissante, l'opinion publique est aussi et surtout un idéal de communauté politique fondée sur la transparence et la publicité. Le principe de publicité suppose un monde où règne une unité entre le peuple et le pouvoir, entre les citoyens et leurs représentants. La sphère politique doit être publique et donc visible aux yeux de tous, comme l'ont montré notamment H. Arendt, C. Lefort et Marc Richir ${ }^{36}$. F. Furet parle en ce sens de l'opinion comme un « miroir des valeurs ${ }^{37} »$.

La publicité met en jeu "l'authenticité des sentiments vertueux" pour reprendre l'expression de Sophie Wahnich ${ }^{38}$. C'est une vertu politique. La publicité est garante, selon Robespierre, de la droiture et de la sagesse ${ }^{39}$. Pour Pétion, seuls les hommes pervers, faibles, corrompus et intrigants la redoutent ${ }^{40}$. Les hommes publics vertueux, au contraire, ne craignent pas d'agir devant son tribunal. L'auteur anonyme du pamphlet Mille et un abus abonde dans le même sens : "L'honnête homme; celui qui se conduit par les principes de l'honneur \& de la probité ne craint jamais le grand jour ${ }^{41}$. »

De vertu on passe ensuite à un critère général de politique : la publicité départage les régimes libres et démocratiques, des régimes despotiques et tyranniques. La transparence garantit la liberté politique. Robespierre oppose ainsi les tyrans et le peuple dans une lutte qui met en confrontation les ténèbres et la lumière : les tyrans « conspirent toujours dans les ténèbres, et [...] le peuple délibère tout haut et au grand jour, sur les moyens de réclamer contre leur tyrannie ${ }^{42} »$. En pleine Terreur, 
Desmoulins rappelle, dans Le Vieux Cordelier, la maxime de Bailly: «la publicité est la sauvegarde du peuple ${ }^{43} »$. Défiant au passage le gouvernement révolutionnaire, qui tente de restreindre la liberté totale de la presse, il ajoute que « la monarchie fait tout dans le cabinet, dans des comités et par le seul secret ; la république, tout à la tribune, en présence du peuple et par la publicité ${ }^{44}$ ».

La problématique de la publicité des procédures parlementaires remonte en fait aux états généraux. En mai 1789, Volney demande par exemple à la chambre du Tiers d'admettre un plus grand public afin qu'elle puisse «s'entourer de l'empire de l'opinion ${ }^{45}$ " qui est, comme nous l'avons vu, l'incarnation de la nation. Lorsqu'en octobre 1789 l'Assemblée nationale transfert son siège au Manège des Tuileries, Marat crie au piège. L'espace est trop restreint pour les spectateurs, qui pourront être plus facilement, affirme-t-il, soudoyés par les aristocrates. Il importe donc "pour le maintien de la liberté que la salle entière contienne au moins deux mille spectateurs, dans des tribunes ouvertes à tous venants, sans distinction ${ }^{46}$. " La publicité créerait en fait un espace de vérité propice à la justice. On le voit très bien avec la proposition de Marat de créer un tribunal d'État pour juger les agents de l'autorité : «la procédure doit toujours être faite en public, parce qu'alors la vérité, n'ayant pas à craindre d'être étouffée par l'intrigue, l'artifice, la violence, peut se montrer dans toute sa pureté, parce que le public est le premier juge des choses qui le concernent ${ }^{47}$ ».

Cette assimilation de la transparence à la vérité et à la justice est également présente dans le discours robespierriste. En février 1792, il propose la construction d'un « édifice majestueux » sur les ruines de la Bastille qui accueillerait l'Assemblée nationale. Le but étant que le "peuple puisse venir commodément et librement entendre discuter ses intérêts et regarder ses mandataires." "Sous les yeux du peuple ", ajoute-t-il, "la pudeur au moins ne permet de trahir audacieusement la cause de la justice et de l'humanitée ${ }^{48}$.» Quelques mois plus tard, dans les premiers jours de la Convention, il juge blasphématoire l'opinion de Lasource qui considère la publicité non pas comme un droit du peuple, mais comme une faveur des représentants. La culture du secret va à l'encontre, selon lui, des « principes». Cette proposition est finalement repoussée par Danton ${ }^{49}$.

La liberté de la presse permet aussi la réalisation de cet espace de transparence auquel peut participer chaque citoyen. En fait, c'est le principal instrument démocratique qui favorise le lien civique. Elle met « toutes les vérités en commun » selon Pétion ${ }^{50}$. Sieyès et Condorcet reprennent l'idée de Malesherbes selon laquelle l'imprimerie serait l'équivalent de la parole du citoyen antique sur la place publique. C'est dans le cadre de son rapport sur les délits de la presse en janvier 1790 que Sieyès reprend cette référence à l'Antiquité : « L'imprimerie est, pour l'immensité de l'espace, ce qu'était la voix de l'orateur sur la place publique d'Athènes et de Rome ${ }^{51}$ ». Heureuse formule récupérée par Condorcet: "Par ce moyen [l'imprimerie] un peuple répandu sur un grand territoire, peut être aussi libre que l'était autrefois le peuple d'une simple cité. Les hommes dispersés peuvent examiner, délibérer, juger comme les hommes réunis [...] C'est par l'impression seule, que les discussions dans un grand peuple, peuvent être vraiment une $[. . .]^{52} »$.

Ce nouveau lien civique établi par l'écrit n'est pas neutre sur le plan politique, il prend une coloration clairement anti-absolutiste. La liberté de la presse s'oppose fondamentalement à toute tyrannie, elle est un gage démocratique comme le soutient Condorcet lors du débat sur la déchéance du roi à l'été $1791:$ «[..] l'usage presque 
universel de la lecture, la multitude de papiers publics, suffisent pour préserver de ce danger ${ }^{53}$. » Le philosophe entend ici démontrer que la grandeur de la France, son vaste territoire, n'est pas un frein à l'établissement de la République et d'un espace public viable. Cette idée n'est pas sans rappeler la belle métaphore de Marie-Joseph de Chénier (été 1789), citée par A. de Baecque :

Une Fée avait doté un prince d'une qualité bienheureuse: c'était de pouvoir entendre et voir, dans le plus grand éloignement, la voix et les corps de son peuple. Sans quitter son trône, il s'instruisait ainsi de la pensée de tous et reconnaissait à leur constitution la misère ou l'opulence de ses sujets. La liberté de la presse tiendra lieu de cette Fée. Car les mots doivent pouvoir aller comme des traits vers leurs cibles, révéler l'injustice et atteindre au cœur des mauvais citoyens, les dissimulateurs de complots contre la nation.

La liberté de la presse est donc fondatrice du politique puisqu'elle lie le pouvoir (le prince) aux « voix » et aux « corps » du peuple au sein d'une « communauté de vision », selon le terme d'A. de Baecque. Elle dévoile également les complots qui pourraient détruire ce lieu commun.

La publicité protège du coup l'espace public et le peuple. Elle fait tomber les masques, révèle l'hypocrisie et dévoile les trahisons, d'où cette obsession révolutionnaire pour la dénonciation de complots ${ }^{54}$. Dénoncer constitue un acte de vérité; c'est dire les choses comme elles le sont véritablement. Or, se masquer ou comploter signifie refuser le monde de réciprocité ouvert par le nouvel espace politique. Les conspirations représentent l'inverse de l'idéal de publicité étant par nature cachées et secrètes. Les conspirations font partie du monde de la «nuit », de l'« ombre ", du «secret » et des "ténèbres". Elles se trament dans des "conciliabules", des «repaires", des "souterrains" et des «assemblées nocturnes ». Les conspirations relèvent ainsi du domaine du non connaissable et du non identifiable, qui échappent aux règles de la nouvelle société démocratique, libre et transparente. Elles se défilent devant le regard du public devenu souverain. "On se cache, on dissimule, on trompe; donc on conspire ", affirme Robespierre au soir de sa vie politique ${ }^{55}$.

\section{Dénonciation, exclusion et domination}

26 La nouvelle culture politique consacre le triomphe de l'opinion publique et légitime ainsi la prise de parole: "everywhere, in short, talk was the order of the day ${ }^{56}$." La libération de l'écrit et de la parole, maintenant à l'ordre du jour, a pour résultat immédiat d'étendre l'espace public. Mais cette extension rapide et maximale n'est pas sans créer de tension au sein du nouvel espace démocratique. Nous limiterons ici notre analyse aux polémiques suscitées par la pratique de la dénonciation et plus particulièrement aux discussions entourant la création des comités de recherches et aux débats sur la guerre, qui montrent les dangers d'une obsession de la transparence publique.

27 Nous avons vu que l'acte de dénoncer constitue l'extension du droit imprescriptible de publier ses opinions. Si elle est un droit, qui découle de la liberté, elle est aussi un devoir afin d'assurer la fondation de cette liberté. En effet, en assurant la transparence publique, la pratique dénonciatrice permet de protéger la nouvelle fondation des dangers de conspirations des « ennemis de la Révolution ». Elle résulte, comme l'a bien souligné C. Lucas, de la prise de conscience de la fragilité des acquis révolutionnaires, constamment menacés par les «ennemis du bien public ${ }^{57} »$. La dénonciation constitue 
donc une arme défensive pour contrer les complots contre la Révolution. En ce sens, elle est, comme le dit Mirabeau et Luchet, le "palladium» de la liberté du peuple ${ }^{58}$. Pour Desmoulins, «la défiance est la mère de sûretés ${ }^{9}$ ». C'est pour cette raison que la dénonciation est vue comme une vertu et un devoir civique.

Cette pratique ne fait toutefois pas consensus. La création des comités de recherche est révélateur des dissensions ${ }^{60}$ qui déstabilisent le nouveau régime. Le Comité de l'Assemblée nationale est créé dans le contexte critique de l'été 1789 marqué par la Grande peur bien étudiée par Georges Lefebvre ${ }^{61}$. Le Comité de la ville de Paris est quant à lui une réponse aux émeutes qui agitent Paris en octobre 1789. Les deux comités ont pour fonction de surveiller l'espace public, en étudiant les diverses délations reçues. Mais comme l'ont montré F. Furet et Patrice Gueniffey ${ }^{62}$, le Comité de l'Assemblée nationale outrepasse son mandat en accaparant des fonctions exécutives, comme la supervision du processus judiciaire et l'utilisation d'informateurs rémunérés sur le terrain. Les représentants de la Commune ont, de leur côté, octroyé des pouvoirs accrus à leur Comité, qui peut appréhender et interroger les suspects.

P. Gueniffey a mis en évidence le fait que deux discours opposés s'affrontent au sujet de la création et de l'action des comités ${ }^{63}$. Plus précisément, deux types de droit ou d'intérêt se confrontent: les droits de la nation et les droits de l'homme ${ }^{64}$. Dans le premier camp, les institutions extraordinaires sont considérées comme nécessaires au salut public, vu comme la loi suprême. Les circonstances justifient les mesures d'exception. La révolution n'est donc pas terminée, des complots existent toujours et menacent la nouvelle fondation politique. C'est alors un devoir moral, comme nous venons de le voir, de prendre des moyens spéciaux pour protéger la liberté naissante. Or, dans le second camp, les comités sont représentés au contraire comme une menace contre les nouvelles libertés individuelles ${ }^{65}$. L'institution est qualifiée d'arbitraire, d' inquisitoriale, de dictatoriale, de tyrannique, de despotique, donc contraire à la démocratie, aux bonnes mœurs, aux principes et à la confiance entre les citoyens ${ }^{66}$. Au lieu d'assurer la sécurité et la tranquillité publiques, les comités sont accusés d'entretenir et d'alimenter la violence populaire, par laquelle on légitime après coup leur existence et leur pouvoir. Ils excitent la haine, ils sèment la terreur et le soupçon ${ }^{67}$.

Par ses fonctions de journaliste et de membre du Comité de recherches, Brissot est amené à défendre à plusieurs reprises les vertus de la pratique dénonciatrice ${ }^{68}$. Mais le débat sur la guerre, dans lequel il occupe une position centrale, le pousse à prendre ses distances vis-à-vis d'une défiance systématique du pouvoir exécutif, telle que pratiquée par ses collègues patriotes. Il plaide au contraire pour un retour de la confiance et pour l'union entre les deux pouvoirs, législatif et exécutif. Cette problématique est au cœur du célèbre duel Brissot - Robespierre qui secoue les tribunes du club des Jacobins de décembre 1791 à janvier 1792 et qu'il convient d'analyser, dans ses grandes lignes, afin de mettre en relief les problèmes liés à la dénonciation sans limite.

Dans le numéro du 14 décembre du Patriote françois, Brissot met en garde ses collègues contre une défiance sans bornes : «La défiance est la première vertu d'un peuple libre ; mais cette défiance doit être raisonnée, car elle devient le plus grand défaut ${ }^{69}$. » Pour prouver cette assertion, il cite l'exemple des « inquiétudes exagérées » et des " fausses alarmes " dans le département de la Gironde qui ont nuit dramatiquement à la circulation des grains. Dans ses trois grands discours au club des Jacobins, Brissot a la possibilité de développer cette thématique afin de rallier et d'unifier les patriotes, $a$ priori méfiants envers une politique souhaitée par le roi, la Cour et le pouvoir exécutif. 
Pour lui, l'« état de défiance est un état affreux » " qui empêche les deux pouvoirs de marcher de concert », ce qui provoque inévitablement la dissolution de "l'empire ${ }^{70}$ ». La confiance mutuelle est donc le mot d'ordre pour pallier ces maux. Brissot ne sousestime pas pour autant les craintes des révolutionnaires. La stratégie de la guerre constitue en réalité une épreuve pour le pouvoir exécutif, qui sera obligé de choisir son camp $^{71}$. Ceci ne veut pas dire qu'il rejette entièrement la pratique. La dénonciation est une arme précieuse qui s'emploie avec parcimonie, soutient-il. Une pratique sans borne donne des résultats contraires aux intérêts du peuple. Elle finit par fortifier le pouvoir ministériel: «Les sauveurs des ministres sont précisément leurs éternels dénonciateurs, ou ceux qui les dénoncent sans preuves $^{72}$. » Il trace les balises pour les véritables dénonciations : «[...] des faits, et point d'hypothèse ; des preuves, et non des soupçons $[. . .]^{73} »$. Une défiance systématique condamne "le gouvernement à une inaction éternelle ${ }^{74} »$.

Le discours du 18 décembre 1791 est l'un des plus connus de Robespierre, souvent interrompu par les applaudissements. Sa position n'est pas pacifique ${ }^{75}$; il ne s'oppose pas en réalité à la guerre. Mais pour bien la mener, il est nécessaire, selon lui, de détruire avant tout les ennemis de l'intérieur ${ }^{76}$. Pour ce faire, il entend dévoiler les «trames funestes ourdies contre la constitution » et légitimer, du coup, la pratique de la défiance, nécessaire pour le salut de la patrie. La guerre est un piège qui vise d'emblée à augmenter la puissance du pouvoir exécutif. La guerre représente donc, dans l'état actuel, une régression de la Révolution; une marche en arrière vers le despotisme et l'aristocratie $e^{77}$. Cette dénonciation est appuyée par une justification de cette pratique. Il demande aux législateurs patriotes de ne point calomnier la défiance, choix « moins affreux [...] que la stupide confiance " proposée par Brissot. La confiance mène directement au " précipice », car elle ne permet pas, selon Robespierre, de sauver la liberté, contrairement à la défiance qui « est la gardienne des droits du peuple. » Le salut de la patrie dépend de cette vertu. Présenter la défiance comme "un état affreux » n'est pas, selon l'Incorruptible, le «langage d'un homme libre ${ }^{78}$ ». En fait, prêcher la confiance ôte «à la liberté sa dernière ressource, la vigilance et l'énergie de la nation ${ }^{79}$ ». La défiance permet au contraire de préparer et d'éclairer la nation contre les éventuelles trahisons du pouvoir exécutif.

Les deux débats - sur les comités de recherche et sur la guerre - révèlent des tensions importantes au sein de la nouvelle culture démocratique. Du débat sur la guerre, Brissot a mis le doigt sur un danger lié à l'abus de la dénonciation: la paralysie du gouvernement et le manque d'unité. En fait, une défiance systématique empêche l'établissement d'une " communauté de vision ", qui relie le pouvoir au peuple. Elle met une distance trop critique qui peut briser le lien de confiance nécessaire au fonctionnement du gouvernement. Or, le principe de publicité supposait un monde uni à travers un miroir de valeurs. Son abus finit par brouiller la transparence.

Du premier débat sur les comités de recherche, on arrive au constat que l'obsession de la transparence peut créer cette fois un climat de méfiance et d'angoisse entre les citoyens eux-mêmes, qui perturbe la tranquillité publique et sape l'espace de réciprocité ouvert en 1789 . Alors que la pratique de la dénonciation publique s'est mise en place grâce à l'ouverture de l'espace public, son institutionnalisation met désormais en danger cet espace, en alimentant la peur, la violence et l'exclusion. Au nom de la sécurité, on passe progressivement d'un espace libre à un espace surveillé. Avec la création des comités de recherche, les révolutionnaires mettent en place les dispositifs 
qui aboutiront à l'établissement des comités de surveillance, puis du comité de sûreté générale, l'un des piliers de la Terreur, qui transformera et finalement pervertira l'idéal de transparence en moyen de police et de domination.

À travers la notion d'opinion publique, les révolutionnaires ont cherché initialement un point d'équilibre entre l'unité et la pluralité. L'opinion publique est à la fois assimilée au pouvoir souverain représentant toute la nation et pensée comme un principe de publicité, assurant la liberté des opinions, donc de la différence entre les citoyens. Or, la frénésie dénonciatrice généralise la suspicion et rompt cet équilibre en réduisant au silence les voix dissonantes, favorisant alors l'uniformisation des opinions.

Le nœud du problème c'est que la transparence est à la fois réclamée au nom des droits individuels et au nom du salut public. C'est ce que Ladan Boroumand a appelé la guerre des principes. À partir des débats parlementaires, elle montre qu'au nom de l'intérêt suprême de la nation, les révolutionnaires en viennent à brimer les droits individuels. Cette guerre dévoile le double visage de la démocratie naissante. Un visage libéral centré sur l'individu, la source du droit. De cette conception découle un pluralisme politique, un droit à la différence garanti par la liberté de penser. Mais il y a aussi un autre visage, qu'on pourrait qualifier de jacobin à la suite des travaux de Lucien Jaume, axé sur la défense de l'être collectif, de la nation en temps de crise ${ }^{80}$. Le droit tire alors son origine première de la nation. "La nation existe avant tout, elle est l'origine de tout ", comme le soutient Sieyès dans son célèbre pamphlet Qu'est-ce que le tiers état ?81 L'unité est fondée dans ce cas sur l'exclusion de l'ennemi, du conspirateur, qui menace l'existence de la communauté nationale. La pratique dénonciatrice, comme droit et devoir, est porteuse de cette double conception du droit, créatrice de tensions lorsque mise dans un contexte de crise et de luttes politiques. Fruit de la libération de la parole, la dénonciation de complot se révèle pourtant être une arme d'exclusion politique qui restreint l'espace public et la liberté d'opinion. Elle s'appuie sur un discours de salut public qui va légitimer le recours à une violence extralégale. La dénonciation de complots participe ainsi à la construction d'une politique de la terreur et d'exception qui peut suspendre les libertés individuelles.

Le débat sur la dénonciation publique met en lumière le double processus identifié en introduction. D'un côté, la pratique dénonciatrice découle de la liberté de la presse. Elle participe à l'ouverture de l'espace public et à l'émergence de ce que P. Rosanvallon a appelé la démocratie d'expression, « qui correspond à la prise de parole de la société, à la manifestation d'un sentiment collectif, à la formulation de jugements sur les gouvernants et leurs actions, ou encore à l'émission de revendications ${ }^{82}$. » C'est la thèse d'Habermas présentée plus haut. D'un autre côté, la défiance a tendance à transformer la question de la transparence en machine politique fabriquant du consensus par l'exclusion, justifiée au nom du salut public. C'est le côté obscur de la démocratie pure analysée par F. Furet à travers la pensée d'A. Cochin. Dénoncer, c'est parler au nom de l'opinion publique, devenue pouvoir. C'est donc exclure en se parant de la légitimité d'une autorité abstraite et souveraine qu'il faut absolument incarner ou plutôt parler en son nom. "Dans ce délire collectif sur le pouvoir» nous dit F. Furet, «la représentation est exclue ou perpétuellement surveillée ${ }^{83} »$. Une fois dans les mains du pouvoir institutionnel, c'est l'espace public qui passera sous surveillance. Le lien 
civique est ainsi fragilisé à la fois verticalement, entre le peuple et le pouvoir, et horizontalement, entre les citoyens eux-mêmes.

Cette problématique qui naît avec la Révolution va hanter tout le $\mathrm{xx}^{\mathrm{e}}$ siècle et même la société d'aujourd'hui. À cet égard, ne pourrait-on pas faire un parallèle entre la double conception de la transparence et les lucioles analysées par Georges Didi-Huberman $?^{84}$ Les lucioles sont ces « lueurs» de contre-pouvoir, de défense des espaces politiques (le débat, la polémique et la lutte), qui s'opposent à la «grande lumière » du pouvoir qui transforme ces espaces en surveillance et en spectacle. Cette réflexion, qui nous mènerait évidemment trop loin, représente une piste de recherche prometteuse, qui relie l'expérience révolutionnaire aux problématiques actuelles de la démocratie...

\section{BIBLIOGRAPHIE}

Anonyme, Les Mille et un abus, (s. 1.), septembre 1789.

Anonyme, Cabale des ministres, Et leur renvoi demandé par le peuple à l'Assemblée nationale, [Paris], [1790].

Aulard François-Alphonse, La Société des Jacobins, Recueil de documents pour l'histoire du club des Jacobins de Paris [1889], t. II, Paris, Jouaust, 1973.

Baecque Antoine de, "La dénonciation publique dans la presse et le pamphlet », in The Press in French Revolution, Oxford, The Voltaire foundation, 1991, p. 261-279.

Baecque Antoine de, Le corps de l'histoire. Métaphores et politique (1770-1800), Paris, Calmann-Lévy, 1993.

Baker Keith Michael, Au tribunal de l'opinion. Essais sur l'imaginaire politique au XVIII' siècle [1990], Paris, Payot, 1993.

Baker Keith Michael, Chartier Roger, « Dialogue sur l'espace public », Politix, vol. 7, n² 26, 1994, p. 5-22.

Brissot de Warville Jacques-Pierre, Lettres à Monsieur le Chevalier de Pange sur une brochure intitulée : Réflexions sur la délation et sur le Comité de recherches, Paris, au bureau du « Patriote françois », avril 1790.

Brissot de Warville Jacques-Pierre, J. P. Brissot à Stanislas Clermont, Sur la diatribe de ce dernier contre les Comités de recherches, et sur son apologie de madame Jumilhac, et des Illuminés, Paris, 28 août 1790.

Brissot de Warville Jacques-Pierre, Réplique de J. P. Brissot à Stanislas Clermont, concernant ses nouvelles Observations sur les Comités de Recherches, sur les causes des troubles, les Folliculaires, le long Parlement d'Angleterre, M. Necker, etc., Paris, Impr. du Patriote françois, 8 octobre 1790.

Brissot de Warville Jacques-Pierre, Discours sur la nécessité de déclarer la guerre aux princes allemands qui protègent les émigrés, prononcé le 16 décembre 1791 à la Société des Amis de la Constitution, Paris, 1791.

Brissot de Warville Jacques-Pierre, Patriote françois, 14 décembre 1791, nº 856. 
Brissot de Warville Jacques-Pierre, Troisième discours sur la nécessité de la guerre, Paris, 20 janvier 1792.

Burnand Léonard, Necker et l'opinion publique, Paris, Honoré Champion, 2004.

Chartier Roger, Les origines culturelles de la Révolution française [1990], Paris, Seuil, 2000.

Clermont-Tonnerre Stanislas de, Réflexions sur l'ouvrage intitulé: Projet de contre-révolution par les somnambulistes, ou Rapport dans l'affaire de MM. d'Hosier \& Petit-Jean ; lu aux comités des recherches de l'assemblée nationale \& de la municipalité de Paris, le 29 juillet 1790, Paris, août 1790.

Clermont-Tonnerre Stanislas de, Nouvelles observations sur les comités des recherches, [septembre 1790], Paris, Desenne, (s. d.).

Condorcet, « De la République, ou un Roi est-il nécessaire à la conservation de la liberté ? », in 1791, Naissance du parti républicain, Paris, EDHIS, 1991.

De Pange François, Lettre à M. de La Harpe, Sur le Comité de Recherches, Paris, 1790.

De Pange François, Réflexions sur la délation, et sur le Comité des recherches, Paris, 1790.

Desmoulins Camille, Discours de la lanterne aux Parisiens, 1789.

Didi-Huberman Georges, Survivance des lucioles, Paris, Minuit, 2009.

Furet François, Penser la Révolution française, Paris, PUF, 1978.

Furet François, « Préface », in Pierre Caillet, Les Français en 1789 : d'après les papiers du Comité des recherches de l'Assemblée constituante (1789-1791), Paris, CNRS, 1991.

Gauchet Marcel, La Révolution des pouvoirs. La souveraineté, le peuple et la représentation, 1789-1799, Paris, Gallimard, 1995.

Godechot Jacques, Les constitutions de la France depuis 1789, Paris, Garnier-Flammarion, 1970.

Gueniffey Patrice, La politique de la terreur. Essai sur la violence révolutionnaire, 1789-1794, Paris, Fayard, 2000.

Guilhaumou Jacques, L'avènement des porte-parole de la République (1789-1792). Essai de synthèse sur les langages de la Révolution française, Paris, Septentrion, 1998.

Habermas Jürgen, L'espace public. Archéologie de la publicité comme dimension constitutive de la société bourgeoise, Paris, Payot, 1993.

Hesse Carla, «La fin de l'Ancien Régime typographique », in Alan Marshall et Thierry Gouttenègre (dir.), L'affiche en Révolution, Vizille, musée de la Révolution française, 1998.

Hunt Lynn, Politics, Culture, and Class in the French Revolution, Berkeley, University of California Press, 1984.

Jaume Lucien, Le discours jacobin et la démocratie, Paris, Fayard, 1989.

Klein Alexandre, Du corps médical au corps du sujet : étude historique et philosophique du problème de la subjectivité dans la médecine française moderne et contemporaine, thèse de doctorat, Paris, Québec, EHESS, université Laval, 2008.

Labrosse Claude, Rétat Pierre, Naissance du journal révolutionnaire, 1789, Lyon, Presses universitaires de Lyon, 1989.

Lapied Martine, Peyrard Christine (dir.), La Révolution française. Au carrefour des recherches, Aix-enProvence, publications de l'université de Provence, 2003. 
Lefebvre Georges, La Grande Peur de 1789, suivi de « Les Foules Révolutionnaires », Paris, Armand Colin, 1988.

Lefort Claude, Essais sur le politique, XIX ${ }^{e}$-XXe siècles, Paris, Seuil, 1986.

Loustalot Élysée, Révolutions de Paris, du 8 au 14 novembre 1789, nº XVIII.

Lucas Colin, "The Theory and Practice of Denunciation in the French Revolution”, Journal of Modern, History, vol. 68, décembre 1996, p. 768-785.

Luchet Jean-Pierre, Les contemporains de 1789 et 1790, ou Les opinions débattues pendant la première législature avec les principaux événements de la Révolution, Rédigé par l'Auteur de la Galerie des ÉtatsGénéraux, Paris, 1790.

Machenaud, Second discours sur la guerre et les moyens de sauver l'État, Société des amis de la constitution, séante aux Jacobins séance du 29 janvier 1792, Paris, Impr. du Patriote français, (s. d.).

Marat Jean-Paul, CEuvres politiques, 1789-1793, texte et guide de lecture établis par Jacques de Cock et Charlotte Goëtz, Bruzelles, Pôle Nord, 1993.

Mirabeau Honoré-Gabriel Riqueti, Lettre du Comte de Mirabeau au Comité des recherches, Paris, 1789. Monnier Raymonde, L'espace public démocratique, Essai sur l'opinion à Paris de la Révolution au directoire, Paris, Kimé, 1994.

Ozouf Mona, L’homme régénéré. Essais sur la Révolution française, Paris, Gallimard, 1989.

Pétion Jérôme, Discours sur la liberté de la presse, Paris, Impr. nationale, 1791.

Rétat Pierre, "L'émergence de l'opinion publique », in L'affiche en Révolution, textes réunis et présentés par Alan Marshall et Thierry Gouttenègre, Vizille, musée de la Révolution française, 1998.

Richir Marc, «Révolution et transparence sociale », préface à J. G. Fichte, Considérations destinées à rectifier les jugements publics sur la Révolution française, Paris, Payot, 1974.

Robespierre Maximilien de, Discours sur le parti que l'Assemblée Nationale doit prendre relativement à la proposition de guerre, prononcé à la Société des Amis de la Constitution, le 18 décembre 1791, Paris, Impr. du Patriote françois, (s. d.).

Robespierre Maximilien de, Discours sur la guerre, 2 et 11 janvier 1792, t. VIII, (s. 1.), [1792].

Robespierre Maximilien de, « Deuxième lettre de M. Robespierre à M. Lafayette sur les lettres de M. Lafayette à l'Assemblée nationale et au roi », Défenseur de la Constitution, fin du mois de juin $1792, \mathrm{n}^{\circ} 7, \mathrm{I}, \mathrm{t} . \mathrm{IV}$.

Robespierre Maximilien de, «Sur la fédération de 1792 », Défenseur de la Constitution, vers le 25 juillet $1792, \mathrm{n}^{\circ} 10, \mathrm{II}, \mathrm{t}$. IV.

Robespierre Maximilien de, Sur les moyens de sauver l'État et la liberté, 10 février 1792, t. VIII.

Robespierre Maximilien de, Contre les factions nouvelles et les députés corrompus, 8 thermidor an II, 26 juillet 1794, t. X.

Robespierre Maximilien de, « Discours sur la liberté de la presse », in Cuvres, Paris, PUF, 1912, t. VII. p. 320-334.

Robespierre Maximilien de, Lettres à ses commettants, t. V, Paris, PUF, 1961.

Rosanvallon Pierre, La contre-démocratie. La politique à l'âge de la défiance, Paris, Seuil, 2006. 
Sieyès Emmanuel-Joseph, Qu'est-ce que le tiers état ?, Paris, PUF, 1982.

Tackett Timothy, Par la volonté du peuple. Comment les députés de 1789 sont devenus révolutionnaires, Paris, Albin Michel, 1997 [1996].

Tocqueville Alexis de, L'Ancien régime et la Révolution, Paris, Garnier-Flammarion, 1988.

Wahnich Sophie, L'impossible citoyen. L'étranger dans le discours de la Révolution française, Paris, Albin Michel, 1997.

Zizek Slvoj, Robespierre : entre vertu et terreur, Paris, Stock, 2008.

\section{NOTES}

1. Ou le «bon plaisir du roi » selon la vieille formule de la monarchie française.

2. Martine Lapied et Christine Peyrard, dir., La Révolution française. Au carrefour des recherches, Aixen-Provence, publications de l'université de Provence, 2003.

3. Jürgen Habermas, L'espace public. Archéologie de la publicité comme dimension constitutive de la société bourgeoise, Paris, Payot, 1993.

4. François Furet, Penser la Révolution française, Paris, PUF, 1978, p. 67.

5. Toute l'entreprise de F. Furet a pour objectif de déboulonner le concept de «révolution bourgeoise " qui implique une nécessité historique et une lecture sociale et économique de la politique. Plusieurs historiens modernistes proches de l'école critique de la Révolution française, comme Keith Michael Baker et Roger Chartier, ont sévèrement critiqué le référent sociologique qui sous-tend l'analyse de J. Habermas, soit la nature bourgeoise de la sphère publique. Keith Michael Baker, Au tribunal de l'opinion. Essais sur l'imaginaire politique au XVIII siècle, Paris, Payot, 1993 [1990] ; "Defining the Public Sphere in Eighteenth-Century France : Variations on a Theme by Habermas", in Craig J. Calhoun (dir.), Habermas and The public Sphere, Cambridge, MIT Press, 1992, p. 181-211; Keith Michael Baker et Roger Chartier, «Dialogue sur l'espace public », Politix, vol. 7, $\mathrm{n}^{\circ}$ 26, 1994, p. 5-22 ; Roger Chartier, Les origines culturelles de la Révolution française, Paris, Seuil, 2000 [1990].

6. François Furet, Penser la Révolution française, p. 272.

7. Voir l'analyse pertinente de Claude Lefort, Essais sur le politique, XIX ${ }^{e}-X X^{e}$ siècles, Paris, Seuil, 1986, p. 71.

8. Cet article reprend, dans ses grandes lignes, le deuxième chapitre de la troisième partie de notre thèse Le pouvoir de l'ombre. L'imaginaire du complot durant la Révolution française, Paris, Québec, EHESS, université Laval, 2008.

9. Antoine de Baecque, Le corps de l'histoire. Métaphores et politique (1770-1800), Paris, Calmann-Lévy, 1993.

10. Alexis de Tocqueville, L'Ancien régime et la Révolution, Paris, Garnier-Flammarion, 1988, p. 45.

11. Sur le concept d'opinion publique avant la Révolution, voir principalement Keith Michael Baker, Au tribunal de l'opinion. Essais sur l'imaginaire politique au XVIII siècle, p. 219-265; Roger Chartier, Les origines culturelles de la Révolution française, p. 37-60 ; Mona Ozouf, L'homme régénéré. Essais sur la Révolution française, Paris, Gallimard, 1989; Léonard Burnand, Necker et l'opinion publique, Paris, Honoré Champion, 2004.

12. Jacques Guilhaumou, L'avènement des porte-parole de la République (1789-1792). Essai de synthèse sur les langages de la Révolution française, Paris, Septentrion, 1998, p. 41.

13. Timothy Tackett, Par la volonté du peuple. Comment les députés de 1789 sont devenus révolutionnaires, Paris, Albin Michel, 1997 [1996], p. 106.

14. Cité d'après Marcel Gauchet, La Révolution des pouvoirs. La souveraineté, le peuple et la représentation, 1789-1799, Paris, Gallimard, 1995, p. 72. 
15. Robespierre, «Discours sur la liberté de la presse », Euvres de Maximilien de Robespierre, Paris, PUF, 1912, t. VII. p. 320-334. Ci-après, auteur, titre, tome et page. Le débat sur la liberté de la presse a lieu les 22 et 23 août 1791 à l'Assemblée nationale. C'est l'occasion pour Robespierre de reprendre une partie de son discours pour combattre le projet du Comité de constitution, rapporté par Thouret, de fixer des bornes légales à la liberté de la presse.

16. Robert, Révolutions de l'Europe et Mercure nationale réunis, journal démocratique, $\mathrm{n}^{\circ}$ 18, p. 572.

17. Certains patriotes, comme Collot d'Herbois, parlent aussi de «juré » pour qualifier l'opinion publique. "Opinion de J. M. Collot d'Herbois sur les coupables démarches du général Lafayette », publiée en juillet 1792 dans Le Défenseur de la Constitution de Robespierre, t. IV, p. 263.

18. «Il s'est élevé un tribunal indépendant de toutes les puissances et que toutes les puissances respectent, qui apprécie tous les talents, qui prononce sur tous les gens de mérite.» Voir l'analyse de Mona Ozouf, L'homme régénéré. Essais sur la Révolution française, p. 34.

19. Citation tirée de l'article de Pierre Rétat, «L'émergence de l'opinion publique », dans L'affiche en Révolution, textes réunis et présentés par Alan Marshall et Thierry Gouttenègre, Vizille, musée de la Révolution française, 1998, p. 80.

20. Jérôme Pétion, Discours sur la liberté de la presse, Paris, 1791, p. 22.

21. Robespierre, «Discours sur la liberté de la presse », p. 321.

22. Machenaud, Second discours sur la guerre et les moyens de sauver l'État, Paris, 29 janvier 1792, p. 22.

23. Anonyme, Cabale des ministres, Et leur renvoi demandé par le peuple à l'Assemblée nationale, [Paris], [1790], p. 7.

24. Si l'émergence de l'opinion publique crée, à partir de 1750, un espace de contestations qui sape le cadre absolutiste de la monarchie française, le pouvoir qu'on lui confère est modelé comme une image substitutive du pouvoir absolu. Voir François Furet, Penser la Révolution française, p. 69 ; Mona Ozouf, L’homme régénéré. Essais sur la Révolution française, p. 35.

25. Jacques Godechot, Les constitutions de la France depuis 1789, Paris, Garnier-Flammarion, 1970, p. 34 .

26. Jacques Guilhaumou, L'avènement des porte-parole de la République (1789-1792). Essai de synthèse sur les langages de la Révolution française, p. 136.

27. Mercure de France, samedi 20 janvier $1790, \mathrm{n}^{\circ}$ 5, p. 381.

28. Antoine de Baecque, Le corps de l'histoire. Métaphores et politique (1770-1800), p. 273.

29. Patriote français, 7 août $1789, \mathrm{n}^{\circ} \mathrm{X}, \mathrm{p}$. 3. Cité par Claude Labrosse et Pierre Rétat, Naissance du journal révolutionnaire, 1789, Lyon, Presses universitaires de Lyon, 1989, p. 195.

30. Archives parlementaires de 1787 à 1860. Recueil complet des débats législatifs et politiques des Chambres françaises. Première série, 1789-1799, Paris, Dupont, puis CNRS, depuis 1867, vol.29, 22 août 1791, p. 632. Ci-après : AP, volume, date, page.

31. Jacques Godechot, Les constitutions de la France depuis 1789, p. 61.

32. Marcel Gauchet, La Révolution des pouvoirs. La souveraineté, le peuple et la représentation, 1789-1799, voir essentiellement la première partie. L'ouvrage de Pierre Rosanvallon sur La contredémocratie s'inscrit largement dans la perspective théorique de M. Gauchet, en montrant notamment que le terme d'opinion publique cherche à répondre à la problématique de la souveraineté du peuple, en lui donnant une «lisibilité» et une «consistance». Pierre Rosanvallon, La contre-démocratie. La politique à l'âge de la défiance, Paris, Seuil, 2006, p. 36.

33. Cité d'après l'ouvrage de Raymonde Monnier, L'espace public démocratique, Essai sur l'opinion à Paris de la Révolution au directoire, Paris, Kimé, 1994, p. 34.

34. Citation tirée de l'ouvrage de Marcel Gauchet, La Révolution des pouvoirs. La souveraineté, le peuple et la représentation, 1789-1799, p. 83-84.

35. François-Alphonse Aulard, La Société des Jacobins, Recueil de documents pour l'histoire du club des Jacobins de Paris, t. II, Paris, Jouaust, 1973 (1889), p. 393-394. 
36. Pour les références à Hannah Arendt et à Claude Lefort, voir Essais sur le politique, XIX ${ }^{e}-X X^{e}$ siècles, p. 72 et 143. Marc Richir, "Révolution et transparence sociale", préface à J. G. Fichte, Considérations destinées à rectifier les jugements publics sur la Révolution française, Paris, Payot, 1974, p. 10.

37. François Furet, Penser la Révolution française, p. 83.

38. Sophie Wahnich, L'impossible citoyen. L'étranger dans le discours de la Révolution française, Paris, Albin Michel, 1997, p. 32.

39. Robespierre, «Deuxième lettre de $M$. Robespierre à $M$. Lafayette sur les lettres de M. Lafayette à l'Assemblée nationale et au roi ", Défenseur de la Constitution, fin du mois de juin 1792, nº 7, I, t. IV, p. 206.

40. Jérôme Pétion, Discours sur la liberté de la presse, p. 20.

41. Anonyme, Les Mille et un abus, septembre 1789, p. 54.

42. Robespierre, «Sur la fédération de 1792 », Défenseur de la Constitution, vers le 25 juillet 1792, n 10, II, t. IV, p. 299.

43. Camille Desmoulins, Le Vieux Cordelier, $\mathrm{n}^{\circ}$ 7, p. 108.

44. Ibid., p. 123.

45. Citation tirée de l'article de Pierre Rétat, «L'émergence de l'opinion publique », p. 78.

46. Jean-Paul Marat, CEuvres politiques, 1789-1793, texte et guide de lecture établis par Jacques de Cock et Charlotte Goëtz, Bruzelles, Pôle Nord, 1993. 5 novembre 1789, n 29, t. I, p. 262. Ci-après : auteur, date, numéro, tome, page.

47. Jean-Paul Marat, 13 novembre 1789, nº 37, t. I, p. 297.

48. Maximilien de Robespierre, Sur les moyens de sauver l'État et la liberté, 10 février 1792, t. VIII, p. 174.

49. Maximilien de Robespierre, Lettres à ses commettants, $\mathrm{n}^{\circ}$ 1, II, t. V, p. 22.

50. Jérôme Pétion, Discours sur la liberté de la presse, p. 30. Dans la même voie, Condorcet dit dans le Patriote françois ( $n^{\circ} 848,6$ décembre 1791), que « la discussion générale et publique conduit à la vérité."

51. AP, vol. 11, p. 260.

52. Citation tirée de l'article de C. Hesse, «La fin de l'Ancien Régime typographique », dans Alan Marshall et Thierry Gouttenègre (dir.), L'affiche en Révolution, Vizille, musée de la Révolution française, 1998, p. 71.

53. Condorcet, « De la République, ou un Roi est-il nécessaire à la conservation de la liberté ? », in 1791, Naissance du parti républicain, Paris, EDHIS, 1991, p. 2.

54. Antoine de Baecque, "La dénonciation publique dans la presse et le pamphlet ", in The Press in French Revolution, Oxford, The Voltaire foundation, 1991, p. 261-279; Lucien Jaume, Le discours jacobin et la démocratie, Paris, Fayard, 1989; Colin Lucas, "The Theory and Practice of Denunciation in the French Revolution", Journal of Modern, History, vol.68, décembre 1996, p. 768-785.

55. Maximilien de Robespierre, Contre les factions nouvelles et les députés corrompus, 8 thermidor an II, 26 juillet 1794 , t. X, p. 570.

56. Lynn Hunt, Politics, Culture, and Class in the French Revolution, Berkeley, University of California Press, 1984, p. 20.

57. Colin Lucas, "The Theory and Practice of Denunciation in the French Revolution", p. 769.

58. Mirabeau, Lettre du Comte de Mirabeau au Comité des recherches, Paris, 1789, p. 4 ; Jean-Pierre Luchet, Les contemporains de 1789 et 1790, ou Les opinions débattues pendant la première législature avec les principaux événements de la Révolution, Rédigé par l'Auteur de la Galerie des États-Généraux, Paris, 1790, p. 243-244.

59. Camille Desmoulins, Discours de la lanterne aux Parisiens, p. 112 [34].

60. François Furet, «Préface », in Pierre Caillet, Les Français en 1789 : d'après les papiers du Comité des recherches de l'Assemblée constituante (1789-1791), Paris, CNRS, 1991, p. 5. 
61. Georges Lefebvre, La Grande Peur de 1789, suivi de "Les Foules Révolutionnaires ", Paris, Armand Colin, 1988.

62. François Furet, "préface ", in Les Français en 1789, p. 9. Patrice Gueniffey, La politique de la terreur. Essai sur la violence révolutionnaire, 1789-1794, Paris, Fayard, 2000, p. 86.

63. Patrice Gueniffey, La politique de la terreur. Essai sur la violence révolutionnaire, 1789-1794, p. 82. Ces pages doivent beaucoup au chapitre IV de cet ouvrage.

64. L'Assemblée nationale se divise en deux camps : le premier légaliste regroupe Camus, Duport, Mirabeau, Bouffler et Virieu; le second préconisant des mesures de salut public est formé de Rewbell, Gouy d'Arcy et Robespierre. Voir AP, vol. 8, p. 273-295.

65. À droite comme à gauche, on retrouve des opposants aux institutions d'exception.

66. Le journaliste Loustalot a insisté sur le climat délétère qui pouvait résulter d'une institutionnalisation de la pratique dénonciatrice. Révolutions de Paris, du 8 au 14 novembre 1789, $\mathrm{n}^{\circ}$ XVIII, p. 8-9.

67. Le chevalier de Pange et le député monarchien Clermont-Tonnerre, qui mènent une dure campagne pamphlétaire contre le Comité de la ville, sont les principaux opposants à défendre cette idée. François De Pange, Lettre à M. de La Harpe, Sur le Comité de Recherches, Paris, 1790 ; Réflexions sur la délation, et sur le Comité des recherches, Paris, 1790. Clermont-Tonnerre, Réflexions sur l'ouvrage intitulé: Projet de contre-révolution par les somnambulistes, ou Rapport dans l'affaire de MM. d'Hosier \& Petit-Jean; lu aux comités des recherches de l'assemblée nationale \& de la municipalité de Paris, le 29 juillet 1790, Paris, août 1790 ; Nouvelles observations sur les comités des recherches, Paris, [septembre 1790].

68. Jacques-Pierre Brissot de Warville, Lettres à Monsieur le Chevalier de Pange sur une brochure intitulée: Réflexions sur la délation et sur le Comité de recherches, Paris, avril 1790 ; J. P. Brissot à Stanislas Clermont, Sur la diatribe de ce dernier contre les Comités de recherches, et sur son apologie de madame Jumilhac, et des Illuminés, Paris, 28 août 1790. Réplique de J. P. Brissot à Stanislas Clermont, concernant ses nouvelles Observations sur les Comités de Recherches, sur les causes des troubles, les Folliculaires, le long Parlement d'Angleterre, M. Necker, etc., Paris, 8 octobre 1790.

69. Jacques-Pierre Brissot de Warville, Patriote françois, 14 décembre 1791, $\mathrm{n}^{\circ}$ 856, p. 685.

70. Jacques-Pierre Brissot de Warville, Discours sur la nécessité de déclarer la guerre aux princes allemands qui protègent les émigrés, prononcé le 16 décembre 1791 à la Société des Amis de la Constitution, séante aux Jacobins, Paris, 1791, p. 6.

71. La loyauté du roi a été mise à rude épreuve depuis l'épisode de Varennes (été 1791).

72. Jacques-Pierre Brissot de Warville, Troisième discours sur la nécessité de la guerre, Paris, 20 janvier 1792, p. 15.

73. Ibid., p. 16.

74. Ibid., p. 13.

75. Erreur historique que plusieurs auteurs ont tendance à faire. Le dernier en date : Slvoj Zizek, Robespierre : entre vertu et terreur, Paris, Stock, 2008, p. 13.

76. Maximilien de Robespierre, Discours sur le parti que l'Assemblée Nationale doit prendre relativement à la proposition de guerre, p. 47.

77. Ibid., p. 52.

78. Maximilien de Robespierre, Discours sur la guerre, 2 et 11 janvier 1792, t. VIII, p. 76-77.

79. Ibid., p. 108-109.

80. Lucien Jaume, Le discours jacobin et la démocratie, voir notamment l'introduction, p. 9-28.

81. Emmanuel-Joseph Sieyès, Qu'est-ce que le tiers état ?, Paris, PUF, 1982, p. 67.

82. Pierre Rosanvallon, La contre-démocratie. La politique à l'âge de la défiance, p. 25.

83. François Furet, Penser la Révolution française, p. 83.

84. Georges Didi-Huberman, Survivance des lucioles, Paris, Minuit, 2009. 


\section{RÉSUMÉS}

Cet article vise à explorer la double trame de la modernité au moment de la Révolution française. Il s'agit en fait d'étudier la tension constitutive de la démocratie naissante qui se noue notamment à travers l'émergence de la figure de l'opinion publique, pensée à la fois comme un idéal de relation citoyenne (la transparence), une forme de pouvoir (la souveraineté populaire) et un espace de liberté (la liberté de la presse). Dès le départ, la démocratie est animée par un double processus, consubstantiel à cette forme politique : un premier processus qu'on pourrait qualifier de libéral, favorisant une extension de l'espace public, et un autre plus sombre, qui produit du consensus et de l'exclusion, en resserrant les limites de la sphère publique.

\section{INDEX}

Mots-clés : démocratie, dénonciation, exclusion, opinion publique, transparence

\section{AUTEUR}

\section{PHILIPPE MÜNCH}

Philippe Münch est docteur en histoire et en études politiques de l'université de Laval (Québec) et de l'EHESS de Paris. De 2008 à 2010, il a été chercheur postdoctoral à l'Institut d'histoire de la Révolution française de l'université Paris I, Sorbonne-Panthéon. Il enseigne l'histoire au Collège Jean-de-Brébeuf de Montréal (Québec). 